\section{Bemerkungen zur Mechanik des Nerven- systems. (Die oxygene, organische, Energie.)}

\author{
Von Professor 0. Rosenbach in Breslau.
}

(Schluss aus No. 44.)

III. Der Mechanismns der Degenerationsznstände peripherer Nerven.

Acceptirt man die Thatsache, dass die Ganglienzellen Accumnlatoren oder eigentlich Transformatoren nud Accnmulatoren zngleich sind, so müssen anch zwei Thatsachen der Nervenphysiologie von anderen Gesichtspnnkten ans als bisher betrachtet werden; es ist dies einmal die Lehre von den sogenannten trophischen Centren nnd zweitens die Lehre von dem Wesen der Leitnng motorischer Reize. Wenn die Nerven nur Leitnngsbahnen wären, d. h. nnr dnrch gewisse Formen kinetischer Energie, die in sie einstrahlen, in gewisse Schwingungen der kleinsten Theilchen geriethen, so wäre eine Degeneration der Bahnea nach Abtrennnng vom Centrnm nicht gat erklärlich; denn was wird mit dem Ansdrncke trophisch äberhanpt erklärt? Wenn man jedoch weiss, dass anch die Nerven nicht Leitnngsbahnen, sondern Maschinen sind, deren labiler (dynamischer) Gleichgewichtsznstand, d. h. ihre Arbeitsthätigkeit, erhalten werden mnss, wenn nicht stabile nnd indifferente Isodynamie der Theilchen, also Stillstand der Maschine nnd Zerfall in ein Molecülaggregat, d. h. Lähmnng nnd Tod eintreten soll, so ist es erklärlich, dass der motorische Nerv, der vom Centrum der Energieanhänfnng des Körpers, dem Rückenmark, abgetrennt ist, peripher degeneriren mnss; nnr so ist es erklärlich, dass Spinalnerven die rom Rückenmark, Gehirnner $r e n$, die vom Mittelhirn abgetrennt sind, entarten. Die Transformatoren, die den motorischen Nerven bilden, können nur ce utrifngal geladen werden und müssen degeneriren, sobald der sie speisende, d. h. ihr labiles Gleichgewicht bewirkende, Nervenstrom nuterbrochen ist; denn da sie ja die oxygene Energie wieder in Mnskelerregnng nnd Bewegnng nmwandeln, so können sie nnr vermittels der Form kinetischer Energie, anf die sie eingestellt sind, dauernd arbeiten. Arbeiten sie aber nicht mehr, so tritt Isodynamie nnd Gewebstod ein. Die normale Innervation vom Centrnm ans befördert nnn den Verbrennnngsprocess, sie liefert gewissermaassen kleinste Fnnken, die die Verbrennnng regnliren nud verstärken. Der motorische Nerv, dessen einziges Kraftreservoir das Räckenmark ist, mnss also nach der Abtrennnng degeneriren; aber anch der Mnskel wird theilweise degeneriren, wenn nach Abtrennng des Kraftreservoirs die Möglichkeit schwindet, in ihm Anslösnngsvorgänge in grösserer Ansdehnnng zn erregen.

Die Trennung des motorischen Nerven vom Rückenmark bringt also nicht hypothetische, trophische Centra in Wegfall, sondern sie bewirkt den Stillstand der Maschine, weil die Anslösnngsvorgänge anfhören, die die Voranssetznng des labilen Gleichgewichtsznstandes jeder organischen Maschine sind. Wenn aber die Maschine ans dem labilen in den stabilen Gleichgewichtsznstand übergeht, so steht sie eben still, nnd wenn ihre Theile dann allmählich in den indifferenten Gleichgewichtsznstand eintreten, so ist sie eben keine Maschine mehr, sondern ein Molecülcomplex, der nicht mehr zum Leben zn bringen ist, denn Leben heisst Arbeit leisten.

Bei den sensiblen Nerven liegen die Verhältnisse etwas complicirter. Wenn wir erwägen, dass das centripetale Nervensystem mit seinen Endapparaten ein Sammler kinetischer Energie von grösster Feinheit nnd Exactheit ist, wenn wir erwägen, dass die Sinuesapparate bereits die geringsten Lichtwellen anffangen nud znr Umwandlnag in Nervenenergie nnd als Anslösnngsvorgänge für nubegrenzte Arbeitsleistnng verwerthen - ein kanm messbarer Lichteindrnck vermag die ganze Snmme der Mnskelarbeit des Körpers anszn. lösen- so ist klar, dass Schntzapparate in besonderer Stärke vorhanden sein müssen, nm die anftreffende kinetische Energie (Erschütternng, Stoss) für die Centralorgane möglichst nnmerklich in die oxygene Energie überznführen oder die Thätigkeit der Apparate, die damit betrant sind, beim Eintreffen nener Energiemengen möglichst wenig zn stören. So ist als erster Hemmnngsapparat, als erstes Reservoir, gewissermaassen als Damm das sensible Wnrzelganglion eingefügt, $\mathrm{nm}$ jedenfalls einen Theil der Energie dnrch Anfspeichernng nnd Ableitnng vom Rückenmark nnd Gehirn -- es findet ja anch eine Verbindnng mit Fasern der vorderen Wnrzeln statt - fernzuhalten. Neben diesem Zwecke dient es aber noch einem anderen, nämlich die Maschinen der_sénsiblen (centri- petalen) Nerren in beständigem Gange zn erhalten. So paradox es klingt, es ist höchst wahrscheinlich, dass in den centripetalen Nerven anch recht nmfangreiche centrifngale Impnlse - and zwar hemmende nud beschlennigende - geleitet werden; denu das, was wir Anfmerksamkeit nennen, ist nichts anderes, als ein starker centrifngaler Impnls, der in sogenanten sensiblen (centripetalen) Nerven geleitet wird nnd diese erst anfnahmefähig macht für die Leitnng feinster Reize. Anfmerksamkeit ist also die Aufhebung der Hemmung, die das Gehirnbeständig anf den Accumulator des Rückenmarks ausübt, nnd erst nach dem Anfhören dieser Hemmnng oder mit der Eröffnnng einer Nebenschliessung znm Gehirn können selbst die schwächsten centripetalen Reize in das Gehirn einstrablen.

Dass wir im Schlafe nicht fühlen nod empfinden - die bewnsste Fmpfindnng tritt doch jedenfalls erst im Angenblicke des Erwachens ein, wie gewisse 'Tranmerlebnisse beweisen - bernht nicht etwa bloss daranf, dass das Grosshirn primär, z. B. durch Ermüdnngsstoffe, nnerregbar wird nnd nur deshalb von peripheren Reizen nnbeeinflusst bleibt, sondern vor allem daranf, dass vom Gehirn während des Schlafs beständig hemmende Impnlse nach der Peripherie gehen, die die Leitung $\mathrm{n}$ a ch dem Gehirn einerseits durch Verschlnss der sensiblen Bahnen (Herabsetznng der Erregbarkeit der peripheren Nerven), andererseits dnrch Verschluss der Eingangspforten (Reiznng der Sphincteren) verhindern. Ich habe bereits in früheren Arbeiten über das Verhalten der Reflexe im Schlafe ${ }^{1}$ ) nnd bei Anfstellnng einer Theorie des Cheyne-Stokes'schen Phänomens ${ }^{2}$ ) anf diese scheinbar paradoxe Erscheinng hingewiesen nnd will hier nur bemerken, dass eben im Schlafe die Maschinen der senciblen Nerven nach Möglichkeit "abgestellt" sind, während der Verschlnss der Sphincteren der denkbar stärkste ist. Während also im wachen Znstande das Grosshirn die höchste Feinheit der sensiblen Leitung dadnrch erzielt, dass es die heminenden Impulse, die es fast beständig darch die Hinterstränge nach der Peripherie sendet, fortfallen lässt (Anfmerksamkeit), bewirkt es im Schlafe das Umgekehrte, indem es die hemmenden Impnlse so verstärkt, dass Reize, die sonst in die centripetalen Bahnen geleitet werden, jetzt nicht znm Gehirn gelangen, weil sie in der Peripherie angesammelt werden. Einen Theil der Energie, die es während des Schlafes an motorischen Impulsen (in den Pyramidenbahnen) erspart, veransgabt es dann zn centrifngalen Impulsen in centripetalen Nerven. Wie stark diese Hemmnng sein mnss, ersieht man ans der Thatsache, dass so viele Personen, namentlich Kinder, die ans festem Schlafe erweckt werden, sich ans Leibeskräften am ganzen Körper zn kratzen anfangen - ein Zeichen dafür, dass sich in den während des Schlafs verschlossenen Bahnen der peripheren Nerven sehr beträchtliche Mengen von Reiz angesammelt haben müssen.

In den sensiblen Nerven gehen also beständig centripetale nnd centrifugale Wellen, und die Arbeit der Maschine der peripheren Nerven wird nach zwei Richtnngen hin angeregt, dnrch kinetische (centripetal geleitete) Energie, die transformirt werden soll, nnd durch oxyge ne (centrifngal geleitete), in Nervenkraft" transformirte Energie, die allein den Tonns, die Labilität der Maschine erhält: Der Reiz also, der die nnthätige Maschine erst zur eigentlichen arbeitenden Maschine macht, da er das labile Gleichgewicht erbält, kommt anch für den sensiblen Nerven vom Centram, und daher muss auch der centripalleitende Nerv degeneriren, wenn er von seinem Kraftreservoir, den Spinalganglien, getrennt ist. Dass anch der zwischen Ganglion nnd Rückenmark gelegene Stnmpf des sensiblen Nerven atrophirt, kann niemanden Wnnder nehmen, da ja die im Stnmpfe befindlichen Maschinchen nicht mehr Energiemengen von der Peripherie her erhalten, also der Functionsatrophie anheimfallen müssen, während der periphere Stnmpf, der noch am Ganglion hängt, natürlich nicht atrophiren wird, da er Impnlse jeder Art empfängt nnd mit dem Kraftreservoir znsammenhängt.

Hier ist noch eine Frage zn berühren, die von grosser Wichtigkeit ist, nämlich die Reizleitnng. Die Wellen der Energie im Gewebe pflanzen sich von den Reservoirs, den Accumulatoren, nach allen Richtnngen hin gleichmässig fort, und nur dort, wo es sich $\mathrm{nm}$ allerfeinste Vorgänge der isolirten Fortleitung der Energie handelt, findet wahrscheinlich eine Fortpflanznng nor in longitndinaler Richtnng, in geschlossenen Bahnen, in isolirten Nervenröhren statt. Von dem Kerne ans pflanzt sich die Erregnng nach der ganzen Umgebnng hin fort, von einer Ganglienzelle anf alle mit ihr in Verbindnug stehenden Nerven, ohne dass gerade ein Fortsatz nőthig ist, der direkt mit einer bestimmten Faser in Verbindnng steht.

1) Zeitschr. f. klin. Med. Bd. 1 p. 358.

2) Ibid. Bd. 1 p. 583. 


\section{Die Mechanik der Degeneration des Rückenmarks.}

Dass aus der eben dargelegten Ansicht von dem Mechanismus der Nervenarbeit auch eine wesentlich veränderte Auffassung der Ursache von Nervenkrankheiten, d. h. der Veränderung der Arbeitsleistung eiuzelner Theile des Nervensystems resultirt, mag hier nur angedeutet werden. Eine Krankheit, wie z. B. die Tabes, die eine Einstellung der Arbeitsleistung eines grossen Theiles der nervösen Transformatoren und Accumulatoren darstellt, kann nur daun entstehen, wenn die Energiebildung, die Umformung und die Aufspeicherung der Energie so wesentliche Veränderungen erfahren haben, dass keine Energie für wesentliche Arbeitsleistung mehr vorhanden ist, weil für ausserwesentliche Arbeit zu viel verausgabt wurde. Die Zeit muss kommen, in der man zur Bildung eines natürlichen Systems der Krankheiten ebenso gelangen wird, wie die Botanik zu ihrem natürlichen Systeme gelangte. Man wird die bisherigen Standpunkte der Eintheilung nach äusserlichen, pathognomonischen Kennzeichen verlassen und sich dazu entschliessen müssen, die solidarpathologischen, humoralpathologischen, ätiologischen und pathologisch-anatomischen Gesichtspunkte bei der Aufstellung der Krankheitsbegriffe zu verlassen, um die Leistung (Functionsveränderung) der arbeitenden Maschine allein ins Auge zu fassen. Von Wichtigkeit ist ja doch nur die Betriebsleistung des Gewebes, und deshalb muss die functionelle Diagnostik, die allein aus dem Maasse der ausserwesentlichen Leistung das Verbältniss der inneren wesentlichen Arbeit der Protoplasmamaschinen zu erschliessen bestrebt ist, an Stelle der bisherigen Diagnostik treten, der es genügt erst die letzten Folgen gestörter Arbeit aus den bereits eingetretenen Veränderungen der Form und Beschaffenheit der Gewebe zu erschliessen. Das Gewebe selbst, das specifische Protoplasma, ist die arbeitende Maschine, deren Gesammtleistung das ist, was wir Körperhaushalt nennen. Die Blutversorgung und die Beschaffenheit der Säfte ist einzig und allein der Ausdruck der Thätigkeit des Gewebes, wenn ja auch nicht geleugnet werden soll, dass auch eine primäre Disposition in der Anlage des Circulationsapparates in gewissem Sinne die Arbeit der Theile beeinflussen kann (angeborene Enge des Aortensystems, Missbildungen). Was für alle Theile gilt, gilt auch für das Nervensystem. Da alle seine Maschinen wesentliche und ausserwesentliche Arbeit leisten, so reguliren sie selbst primär den Blutzufluss und gehen zugrunde, nicht weil der Blutzufluss durch primäre Gefässerkrankung stockt - dies kommt nur unter den seltensten Bedingungen (bei Embolieen) vor - sondern weil sie durch zu grosse Ausgaben für ausserwesentliche Arbeit eine Verringerung der zu ihrem eigenen Betriebe nöthigen Energie erfahren und somit unter Einschränkung des Betriebes allmählich zur minimalsten Leistung gelangen, ja der Zerstörung anheimfallen müssen, weil sie ja die zu ihrem Betriebe nöthige Energie, die oxygene Kraft nur aus ihrer vollen Arbeit gewinnen können.

Den Versuch, die Tabes als primäre Gefässerkrankung aufzufassen, können wir ebenso wie den Versuch, sie als alleiniges Product der Lues hinzustellen, nur als einen böchst unbefriedigenden bezeichnen; denn Gefässe können nicht primär erkranken, da die Arbeitsleistung des Blutgefässsystems nur der Ausdruck seiner specifischen Gewebsenergie ist. Je mehr das Gewebe (auf irgend welche Reize hin) arbeiten muss, desto grösser ist auch der Blutzufluss, wie aus der obigen Auseinandersetzung über die Thätigkeit der Protoplasmamaschine hervorgeht; aber je mehr chemische (wesentliche und ausserwesentliche Arbeit) das Gewebe leisten muss, desto geringere mechanische kann es leisten und umgekehrt. Je mehr Arbeit dem Nervenorgan schon an und für sich bei Umsetzung der Energie (bei der wesentlichen Arbeit) entsteht, desto schwieriger und ungenügender leistet es seine ausserwesentliche Arbeit, die Reizleitung und Reizumformung, die Innervation, und je mehr Energie es zur Ueberwindung der Widerstände braucht, desto schneller erschöpft sich sein Energievorrath. Die Tabes kann also nur auf Grund der Erschopfung der Nervenkraft entstehen, und diese ist entweder das Resultat $\mathrm{zu}$ geringer Fähigkeit, Energie zu bilden, oder zu grosser (absoluter oder relativer) Ausgaben an Nervenenergie. Je grösser diese ausserwesentliche Leistung der Maschine ist, je mehr Energie für die (äussere) Arbeitsleistung verausgabt wird, desto weniger kann die zur Auslösung der Vorgänge nöthige Energiemenge ersetzt werden, und der Effect ist Verringerung der Auslösungsvorgänge, Verringerung der Arbeit der vom Nervensystem erregten Theile und geringerer Zufluss und verminderte Bildung der Energie, die in Nervenenergie umgeformt werden soll.

Die Lues als Ursache der Tabes anzusehen, ginge nur dann an, wenn bewiesen würde, dass das luetische Virus eine besondere Vermehrung der ausserwesentlichen und wesentlichen Arbeit des Rückenmarks nöthig machte, etwa wie Strychnin oder andere Gifte; dann wäre die Erschöpfung der Energie, der Stillstand und die schliessliche Vernichtung der Nervenmaschine erklärlich.
Als Ursache der Tabes muss also unserer Ansicht nach vor allem Erkältung angeschuldigt werden, da sie die Wärmebildung in den Muskeln, die Hauptquelle der zur Aufnahme und Transformation gelangenden Wärme, verhindert und vielleicht auch direkt auf die Arbeit der Nervenmaschinen der Peripherie einen ungünstigen Einfluss übt. Die Abkühlung der unteren Extremitäten muss in dieser Beziehung von besonderer Bedeutung sein, da gerade die von den unteren Extremitäten kommende Wärme ja zur Bildung der oxygenen Energie in den unteren Rückenmarksabschnitten vorzugsweise nöthig scheint. Als fernere Ursachen für die Entstehung der tabischen Störung können andauernde starke Muskelanstrengungen (namentlich in feuchter und kalter Umgebung) und besonders auch starke und langandauernde sensible Erregungen etc. in Betracht kommen, da sie besondere Ausgaben an Energie nöthig machen und zudem die Arbeit der Maschine wesentlich erschweren. Jedenfalls aber muss auch hier das Bestehen einer angeborenen Disposition, einer Schwäche der Anlage, die selbst relativ nicht grosse Ausgaben an Nervenenergie nicht wieder zu ersetzen gestattet, in Betracht gezogen werden.

In einem folgenden Artikel soll das Wesen der Reflexbewegung und die Mechanik der Innervation im Gehirn unter normalen Verhätnissen, im Schlafe und im Coma erörtert und namentlich dargelegt werden, wie die Umformung der Energie in bestimmten Apparaten zur Ausbildung von Vorstellungen und zu einer Einwirkung dieser letzteren auf die sogenannte motorische Innervation führen kann. Es soll gezeigt werden, dass das Gehirn nicht eigentlich das Organ der Erregung (der Reizung), sondern das der Hemmung ist, und dass der Vorgang, den wir als motoris che Innervation vom Gehirn aus bezeichnen, nur dem Fortfall einer vom Gehirn beständig ausgeübten Hemmung und der Eröffnung einer Nebenschliessung zur eigentlichen Hauptbahn entspricht, welch' letztere von der Peripherie zum Rückenmarksaccumulator und von diesem durch das Rückenmark zu dem Muskel führt. Der motorische cerebrale Impuls ist der Fortfall der normalen Hemmung, die die Rückenmarksaccumulatoren in der grauen Substanz an der beständigen Bildung eines nach den Muskeln fliessenden starken Stromes hindert. Es soll ferner gezeigt werden, dass auch die gebräuchliche Nomenclatur der Gehirn- und Rückenmarksbahnen recht deutlich die missverständliche Auffassung der psychomotorischen Thätigkeit documentirt, die die ganze neuere Theorie der Localisation im Gehirn und die Lehre von den Centren des Gehirns beherrscht. Man kann es in der That doch nur als eine Verirrung bezeichnen, wenn gewissen Bahnen die Bezeichnung der "transcorticalen“ beigelegt wird; denn nur eine durchaus missverständliche Auffassung von dem Verhältnisse der Psyche zum Gehirn und zu den sogenannten Centren kann den Grund dafür abgeben, dass man in dem Bestreben, die höchsten Centra für eine bestimmte Form der Innervation zu finden, die letzten bekannten Verbindungen des Reflexbogens - die anatomisch als Endstationen der Innervation zu bezeichnenden Ganglien der Grosshirnriude - eben noch nicht als letzte körperliche Station ansehen will, obwohl es eben aus räumlichen Gründeu doch keine weiteren Centra jm Organismus geben kanu. Nur das unklare Bestreben, keinen geschlossenen Reflexbogen, wie ihn die räumliche Anordnung der Gehirnmaterie erfordert, anzunehmen, hat zur Aufstellung des Begriffes der transcorticalen Bahnen geführt, eines Begriffes, der ebenso gut durch die Bezeichnung transcendentale Bahnen" ersetzt werden könnte; denn über den Cortex hinaus giebt es eben kein anatomisches Organ mehr, also auch keine Bahnen im materiellen Sinne. Die Bahnen, die die einzelnen Organe des Cortex verbinden (die Associationssysteme), können nur als intercorticale Bahnen bezeichnet werden, und das Organ, der Stoff, den wir als Psyche bezeichnen, kann nicht über den corticalen Centren stehen, sondern muss sich zwischen ihnen befinden. Das psychische Organ muss aus einer Materie bestehen, die wir zwar nicht sehen können, aber die an die sichtbare Materie geknüpft ist, wie das, was wir als "Kraft" (Energie) bezeichnen, an die Materie geknüpft und nur die Aeusserung einer besonderen Form der Materie ist. Das Grosshirn, als Organ der Psyche, ist in den Körper eingeschaltet, an den Körper, dessen motorisches Innervationscentrum das Rückenmark ist, angeschlossen, besitzt aber weder direkte sensorische, noch motorische Bahnen. 